\title{
CCDC114, DNAI2 and TOP2A involves in the effects of tibolone treatment on postmenopausal endometrium
}

Yanhua $\mathrm{Lv}^{1 \dagger}$, Yanqing Liu ${ }^{2 \dagger}$, Yueqiang Wang ${ }^{3}$, Fanrong Kong ${ }^{1}$, Qiuxiang Pang ${ }^{4}$ and Guirong $\mathrm{Hu}^{5^{*}}$

\begin{abstract}
Background: This study aimed to explore the molecular mechanisms of tibolone treatment in postmenopausal women.

Methods: The gene set enrichment profile, GSE12446, which includes 9 human endometrial samples from postmenopausal women treated with tibolone (tibolone group) and 9 control samples (control group), was downloaded from GEO database for analysis. Differentially expressed genes (DEGs) in tibolone vs. control groups were identified and then used for function and pathway enrichment analysis. Protein-protein interaction (PPI) network and module analyses were also performed. Finally, drug-target interaction was predicted for genes in modules, and then were validated in Pubmed.
\end{abstract}

Results: A total of 238 up-regulated DEGs and 72 down-regulated DEGs were identified. These DEGs were mainly enriched in various biological processed and pathways, such as cilium movement (e.g., CCDC114 and DNAI2), calcium ion homeostasis, regulation of hormone levels and complement/coagulation cascades. PPI network contained 368 interactions and 166 genes, of which IGF1, DNALI1, CCDC114, TOP2A, DNAH5 and DNAI2 were the hue genes. A total of 96 drug-gene interactions were obtained, including 94 drugs and eight genes. TOP2A and HTR2B were found to be targets of 28 drugs and 38 drugs, respectively. Among the 94 obtained drugs, only 12 drugs were reported in studies, of which 7 drugs (e.g., epirubicin) were found to target TOP2A.

Conclusions: CCDC114 and DNAI2 might play important roles in tibolone-treated postmenopausal women via cilium movement function. TOP2A might be a crucial target of tibolone in endometrium of postmenopausal women.

Keywords: Differentially expressed genes, Function and pathway analysis, Gene-drug investigation, Postmenopausal women, Protein-protein interaction network

\section{Background}

The emergence of various characteristics of metabolic syndrome are implicated in the process of premenopause transition to postmenopause [1]. As women age and estrogen levels decrease, coronary artery disease,

\footnotetext{
*Correspondence: nwp848pwspzt0@sina.com

${ }^{\dagger}$ Yanhua Lv and Yanqing Liu have contributed equally to this work

${ }^{5}$ Department of Obstetrics and Gynecology, People's Hospital of Jiaxiang

County, No. 188 Yingfeng Road, Jiaxiang, Jining 272400, Shandong, China

Full list of author information is available at the end of the article
}

osteoporosis, and endometrial diseases are major causes of mortality and morbidity [2,3]. Observational studies have suggested that postmenopausal hormone treatments, such as estrogen replacement therapy, are effective in preventing endometrial disease [4]. However, a previous study found that while estrogen treatment reduced the occurrence of heart disease and fractures in postmenopausal women, long-term treatment with estrogen promoted the risk of endometrial cancer [5]. 
Unopposed estrogen treatment raises the risk of endometrial hyperplasia and the succeeding carcinoma [6].

Tibolone is a synthetic steroid used for hormone replacement therapy in postmenopausal women [7]. Tibolone shows tissue-selective properties that have estrogenic activity in the vagina, brain, and bone, but not in endometrial and breast tissues [8]. Tibolone does not stimulate the endometrium and does not induce endometrial hyperplasia in postmenopausal women. In comparison with estrogen-progestogen therapy, tibolone is associated with less vaginal bleeding $[9,10]$. It had been reported that low doses of tibolone had favorable effects on postmenopausal endometrium by inducing progestogenic properties to create a balance between pro- and anti-apoptotic actions [11]. Nevertheless, there is a requirement to further investigate the molecular mechanisms of action of tibolone in postmenopausal endometrium.

Hanifi-Moghaddam et al., generated the GSE12446 microarray dataset, which compares gene expression between endometrial samples from postmenopausal women after treatment with tibolone for 21 days and endometrium from women treated with estradiolonly + medroxyprogesterone acetate [12]. They observed a close relationship between tibolone treatment and gene expression in endometrium from both groups of women. However, the study did not examine the detailed molecular mechanisms of tibolone action in postmenopausal women.

Herein, we used gene expression data in GSE12446 (of the 36 samples, only data from 9 tibolone-treatment samples and 9 control group samples were used) to perform bioinformatics analysis to further investigate the molecular mechanisms of tibolone action in postmenopausal endometrium [12]. Differentially expressed gene (DEGs) were identified and used in function and pathway enrichment analysis and protein-protein interaction (PPI) network analysis. Finally, drug-gene interactions prediction was performed to identify drugs with potential for use in postmenopausal women. The current study will provide a theoretical basis for further research regarding tiboloneinduced gene expression changes in the endometrium of postmenopausal women, and provide potential targets for use in clinical treatment (Additional file 1: Figure S1 shows the workflow of this study).

\section{Methods}

\section{Microarray data}

The microarray dataset "GSE12446" was downloaded from the Gene Expression Omnibus (GEO, http://www. ncbi.nlm.nih.gov/geo/) database. The samples were pure endometrial tissue collected from patients who underwent vaginal hysterectomy for treatment of prolapse.
Of the 36 samples in total, the gene expression data of 9 samples from the tibolone group $(2.5 \mathrm{mg}$ oral tibolone administered daily, starting 21 days prior to surgery) and 9 samples from the control group (no hormonal treatment) were used. The microarray platform used was the GPL570 (HG-U133_Plus_2) Affymetrix Human Genome U133 Plus 2.0 Array.

\section{Data processing and DEGs screening}

CEL raw data was preprocessed based on a robust multiarray average (RMA) method of affy (version: 1.50.0) package in R software [13]. Probe IDs were converted to gene symbols based on the annotation files from the platform. The probes that did not match with a gene symbol were removed, and the mean value was selected as the final expression value when multiple probes matched to only one gene symbol. Differential gene expression analysis between tibolone-treated and control group samples was performed using the Bayesian method in Linear Models For Microarray Data (limma) [14]. The Benjamini and Hochberg method was used to perform multiple testing correction. Adjusted $P$ value (adj.P.Value) $<0.05$ and $\log$-fold change (FC) $\geq 1$ were selected as the threshold values for DEGs screening. Screened DEGs were then visualized using heat maps and volcano plots.

\section{Function and pathway enrichment analysis}

DAVID software (version: 6.8, https://david-d.ncifcrf. gov/) [15] was used to examine enriched functional pathways in the screened DEG lists based on gene ontology (GO) annotations [16] and Kyoto Encyclopedia of Genes and Genomes (KEGG) pathways [17]. Significantly enriched GO terms and KEGG pathways were defined using a threshold of $P<0.05$ and enriched gene count $\geq 3$.

\section{PPI network construction and module analysis}

To explore interactions between proteins encoded by DEGs, DEGs were uploaded to the STRING database (version: 10.0) [18] to extract PPIs using parameters set to: median confidence $($ score $)=0.4$, species $=$ homo. The resulting PPI networks were visualized using Cytoscape (version: 3.4.0). CytoNCA software [19] was used to analyze the topology properties of nodes in the networks. MCODE (Version1.5.1) [20], a plug-in of Cytoscape software, was used to conduct cluster analysis and identify functional modules from the PPI network using default parameters. Functional modules with a modules score $\geq 5$ were considered significant. ClusterProfiler (version: 3.8.1) software was used to further investigate genes in significant functional modules for enriched biological processes using GO annotations and KEGG pathways. The Benjamini and Hochberg method was used to perform multiple testing correction. The significantly 
enriched terms were selected with the cut-off of adj.P. value $<0.05$ and count $\geq 2$.

\section{Prediction of drug-gene interactions}

Based on the online GDIdb [21]database (version: 3.0), the gene-drug interactions were predicted based on the genes in significant modules using the following parameter settings: Preset Filters, FDA Approved; Advanced Filters, Source Databases, 20 of 20; Gene Categories, 41 of 41; Interaction Types, 51 of 51. Drug-gene interactions were visualized using Cytoscape software. These drug-gene interactions were further validated by searching Pubmed using the key words of 'Postmenopausal', and 'Endometrium,' and "drug names" using Biopython in python software.

\section{Results}

\section{DEGs between tibolone group and control group}

A total of 238 significantly upregulated DEGs and 72 significantly down-regulated DEGs were identified in the tibolone group compared to the control group $(P<0.05$, $\geq$ twofold change, Fig. 1a) and used for subsequent analysis. As shown in heatmap of DEGs, the samples in different groups could be obviously distinguished by upregulated and down-regulated genes. A volcano plot of the screened DEGs is shown in Fig. 1b.

\section{Enrichment analysis of DEGs}

The DEGs were significantly enriched in 36 GO-biological processes and 3 KEGG pathways in the functional enrichment analysis (Additional file 2: Table S1). The three KEGG pathways and the top 10 GO-biological processes were shown in Fig. 2. These DEGs were predominantly involved in functions like cilium movement (GO: 0003341, Genes: Coiled-Coil Domain Containing (CCDC) 114, Dynein Axonemal Intermediate Chain (DNAI) 2, etc.), cilium assembly (GO: 0042384, Gene: CCDC113, DNAI2, etc.) and microtubule-based movement (GO: 0007018, Gene: Dynein Axonemal Heavy Chain (DNAH) 9, Kinesin Family Member (KIF) 4A, etc.). Pathway analysis showed that these DEGs were enriched in pathways including Huntington's disease (hsa04610), systemic lupus erythematosus (hsa05016) and complement/coagulation cascades (hsa05322).

\section{PPI network and module investigation}

To further derive effective information from the identified DEGs, PPI networks were constructed based on the relationships among proteins that the DEGs are known to encode. A total of 368 protein interactions and 166 nodes were revealed (Fig. 3a). In the network topological properties analysis, the degree of each node were analyzed (Additional file 3: Table S2). It could be seen that insulin like growth factor 1 (IGF1, degree=17), DNALI1 (degree $=16)$, CCDC114 $($ degree $=15)$, DNA topoisomerase II alpha (TOP2A, degree $=14$ ), DNAH5
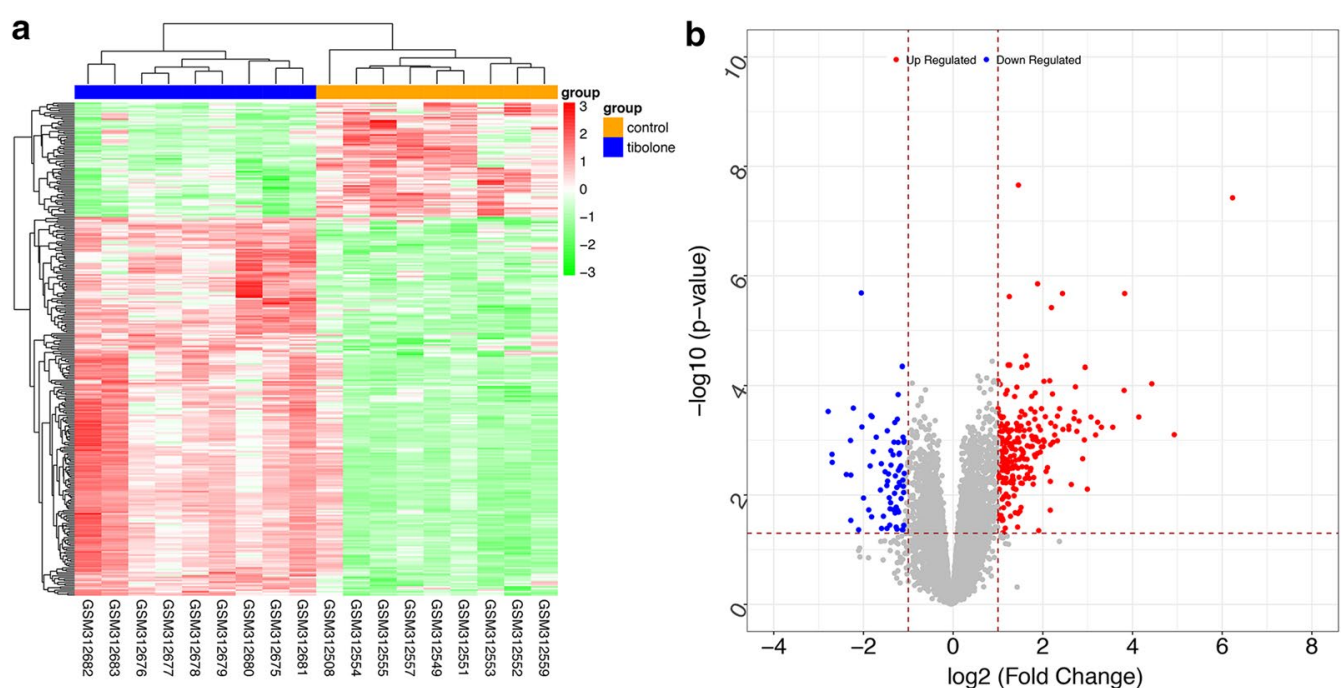

Fig. 1 Heat map and volcano plot of differentially expressed genes in tibolone-treated samples compared to untreated control samples of endometrium from postmenopausal women. a Heat map of DEGs. Yellow bar represents control samples, blue bar represents tibolone treatment samples, green represents down-regulated genes, red represents up-regulated genes. Darker color shades represent greater statistical significance. $\mathbf{b}$ Volcano plot of DEGs. Blue nodes represent down-regulated genes, red nodes represent up-regulated genes, grey nodes represent non-differentially expressed genes. The $x$-axis represents log fold-change values, the $y$-axis represents -log $P$ values 


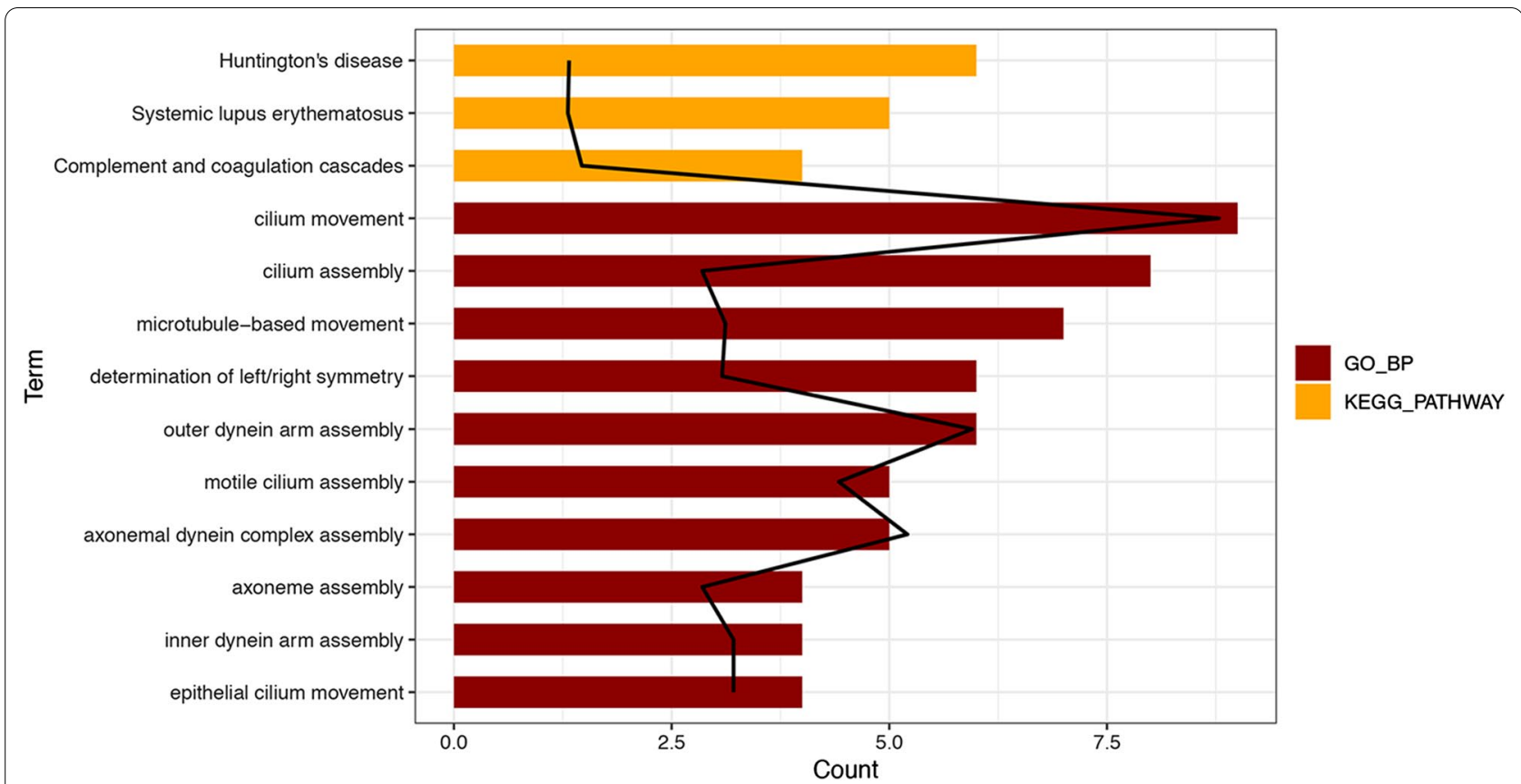

Fig. 2 Function and pathway enrichment analysis of the differentially expressed genes. Enrichment analysis indicating the biological function and pathways likely involved based on DEGs in response to tibolone in postmenopausal endometrium. Dark red represents the top 10 functions; yellow represents the top 3 pathways. Black lines represent $-\log P$ values. The $x$-axis represents the number of genes in a certain block; the $y$-axis lists the function or pathway

(degree $=13)$, DNAI2 (degree $=13)$ and Kinesin Family Member 11 (KIF11, degree $=13$ ) were hub nodes with higher degree in PPI network.

Furthermore, three significant modules with score $\geq 3$ were identified from PPI network(Fig. 3b-d). Module 1 contained 18 up-regulated genes, including TOP2A, KIF11, CCDC114 and DNAI2, etc. The genes in Module 1 were significantly enriched in 14 GO terms and only one KEGG pathway, such as microtubule-based movement (GO: 0007018), mitotic cytokinesis (GO: 0000281) and Huntington's disease (hsa04610). Module 2 contained 6 DEGs (ANAPC4, HIST2H2BD, HIST2H2BE, etc.), and these genes were mainly enriched in $7 \mathrm{GO}$ terms and 4 KEGG pathways, such as nucleosome assembly (GO: 0006334), protein-DNA complex assembly (GO: 0065004), and pathways like systemic lupus erythematosus pathology (hsa05016). Module 3 contained 5 DEGs, and these DEGs were predominantly enriched in $24 \mathrm{GO}$ terms and 7 KEGG pathways, such as calcium ion homeostasis (GO: 0055074), regulation of hormone levels (GO: 0010817), gap junction pathways (hsa04540), and calcium signaling pathway (hsa04020) (Fig. 4, Additional file 4: Table S3).

\section{Drug-gene interaction investigation}

Drug-gene interactions were predicted for the 29 genes in significant modules. A total of 96 drug-gene interactions were obtained, including 94 drugs and eight genes (Fig. 5, Additional file 5: Table S4). Among the eight genes, there were three down-regulated genes and five up-regulated genes, including ribonucleotide reductase regulatory subunit M2 (RRM2), TOP2A, PDZ binding kinase (PBK), and 5-hydroxytryptamine receptor 2B (HTR2B). TOP2A was found to target by 28 drugs and HTR2B was a target of 38 drugs. These interactions included 28 inhibitor relationships, 21 antagonist relationships, 10 agonist relationships, 4 agonist or antagonist relationships, 1 binder relationship, 1 binder/ antagonist relationship, 1 partial agonist relationship and 30 unknown relationships. Such as, epirubicin was predicted to be an inhibitor for TOP2A, and eletriptan was predicted to be an agonist for HTR2B.

\section{Validation of drugs}

The obtained drug-gene interactions above were further validated by searching Pubmed using the key words of 'Postmenopausal', and 'Endometrium,' and "drug names" using Biopython in python software. Among the 94 drugs, only 12 drugs were reported in studies (Fig. 6, Additional file 6: Table S5), including epirubicin, Tretinoin, Digoxin, Paclitaxel, Podofilox, Doxorubicin, Daunorubicin hydrochloride, Daunorubicin, Acetic acid, Vincristine, Cyclophosphamide and Gonadotropin chorionic. 

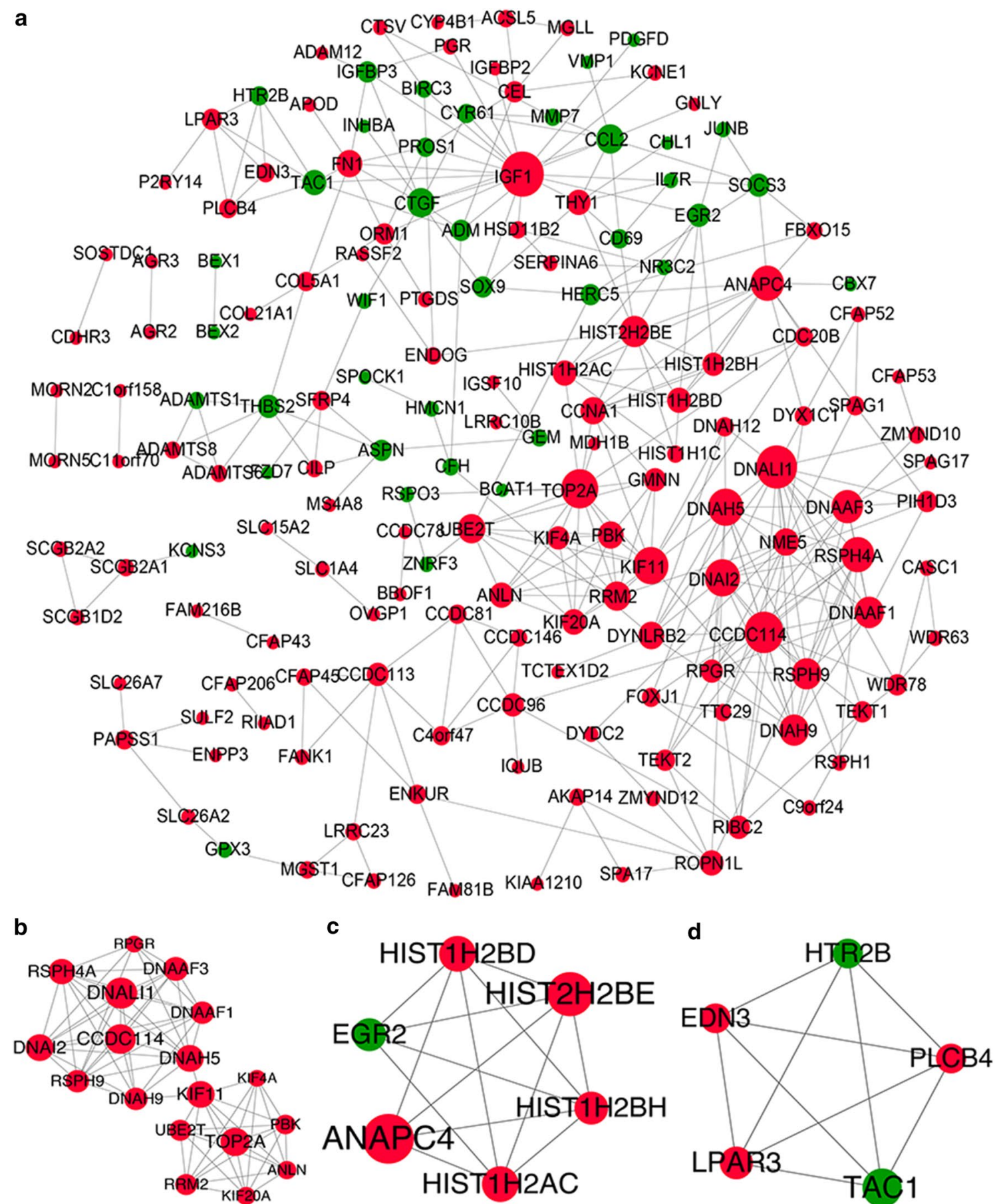

Fig. 3 Protein-protein interaction network and modules. PPI network analysis shows the known and predicted interactions (including physical and functional associations) between proteins encoded by DEGs. Theses connectivity networks are helpful in fully understanding biological phenomena a PPI network. b Module 1. c Module 2. d Module 3. Red circles represent up-regulated genes; green circles represent down-regulated genes; the larger the node size, the higher the degree value. Lines between two nodes represent an interaction

\section{Discussion}

Although tibolone is commonly used as an alternative for estrogen replacement therapy, the detailed molecular mechanism of action of tibolone and its clinical application in postmenopausal women are still unclear. In the present bioinformatics study, a total of 238 up-regulated 


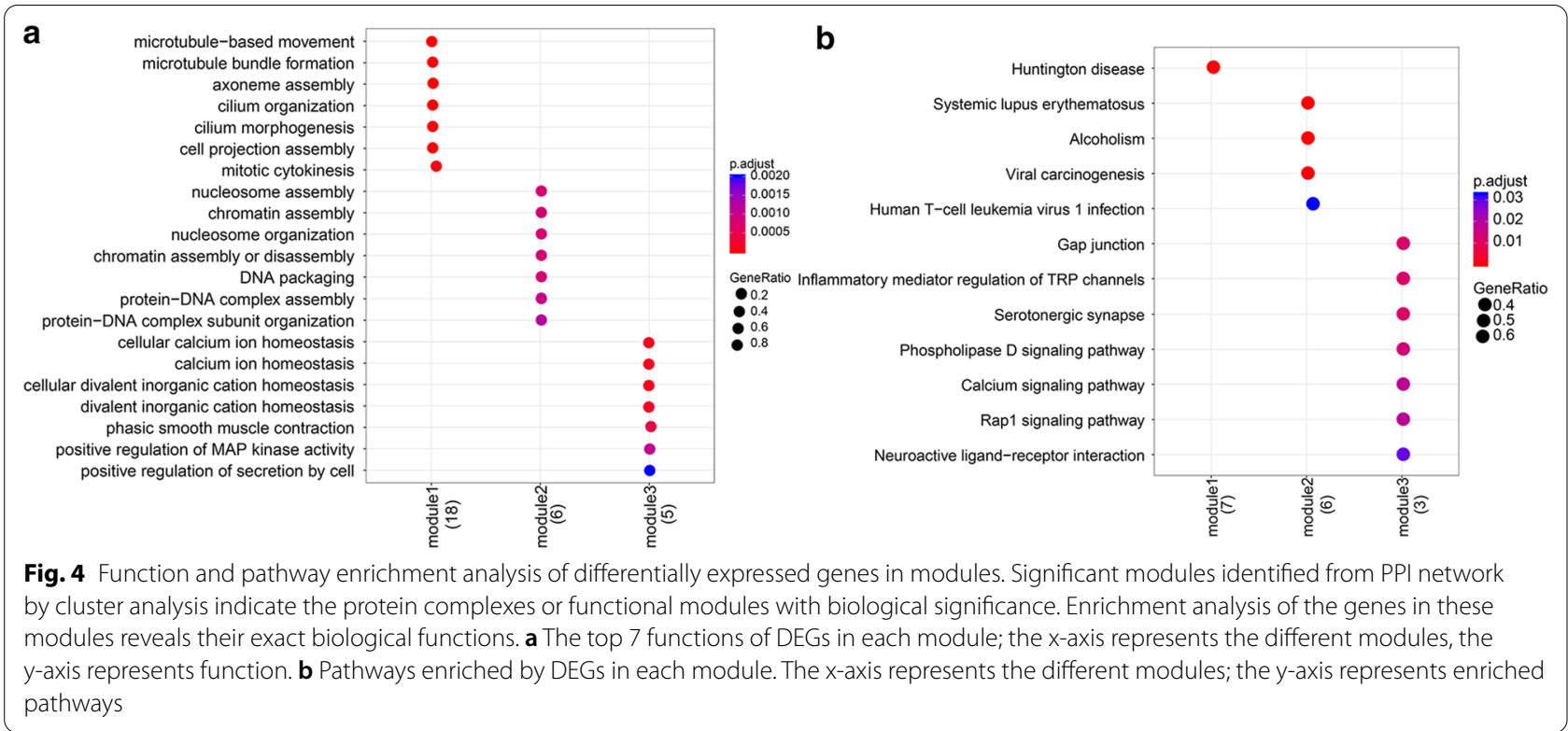

DEGs and 72 down-regulated DEGs were identified and investigated. These DEGs were predominantly assembled in functions such as cilium movement, calcium ion homeostasis, regulation of hormone levels, complement/ coagulation cascades, etc. IGF1, DNALI1, CCDC114, TOP2A, DNAH5 and DNAI2 were the hue genes in PPI network. Furthermore, TOP2A and HTR2B were found to be targets of 28 drugs and 38 drugs, respectively. Among the 94 obtained drugs, only 12 drugs were reported in studies, of which 7 drugs (for example, epirubicin) were found to target TOP2A. These suggested that TOP2A might be a crucial targets in endometrium of drug treated in postmenopausal women.

Cilium movement is closed related to the biological functions of the human oviduct [22]. The frequency of cilium movement appears to increase in the late follicular phase compared to that in both the early follicular phase and the luteinizing phase, and can be reduced by progesterone receptor regulation [23]. Human endometrium consists of stromal and epithelial cells, of which, epithelial cells include secretory and ciliated cells. Ciliogenesis in endometrium is driven by estrogen signaling [24]. Changes in the expression of cilia-related genes has been reported to be associated with differentiation, nodal metastasis and recurrence of endometrial cancer [25].

Cilium-associated CCDC114 participates in cilium movement function in vivo [26]. Mutations in CCDC114 are a cause of primary ciliary dyskinesia (PCD) [27]. A mutation in the CCDC114 gene causes PCD with normal fertility in humans [28]. Although CCDC114 has been shown to be essential for motile cilia in many diseases, its effects in postmenopausal women has not been well studied. DNAI2 mutation is another important factor influencing the function of cilium movement [29]. DNAI2 is down-regulated in the oviduct of non-obese diabetic mice when compared with that in healthy mice [30]. In mouse ovaries, the expression of DNAI2 was detected at high levels in vivo on day 10, with a subsequent decrease on days 15 and 20 [31]. In the current study, GO functional analysis of DEGs identified cilium movement function as an enriched pathway that both CCDC114 and DNAI2 were up-regulated in. Thus, we speculated that CCDC114 and DNAI2 may have a role in cilium movement function in postmenopausal women treated with tibolone.

Epirubicin (4'-epi-doxorubicin) is an anthracycline antibiotic, a doxorubicin analog differing by epimerization of the hydroxyl group at position $4^{\prime}$ of the aminosugar moiety. Similar to doxorubicin, epirubicin shows anti-tumor activity by binding to DNA and inhibiting DNA synthesis and function [32, 33]. Epirubicin has been used as a first-line medicine for cancer in postmenopausal women [34]. Although there is a dose-response effect for epirubicin in clinical treatment for postmenopausal patients [35], the combination of epirubicin and other drugs, including docetaxel and tamoxifen, can enhance the effects of epirubicin [36, 37]. However, whether epirubicin is suitable for the treatment of postmenopausal women is still unclear due to multiple drug resistance [38]. Interestingly, the expression of the TOP2A gene is closely related to epirubicin and drug-resistance [39]. TOP2A alterations are a predictive marker for epirubicin sensitivity in clinical treatment [40]. TOP2A protein levels are used as a predictor of response to epirubicin as 


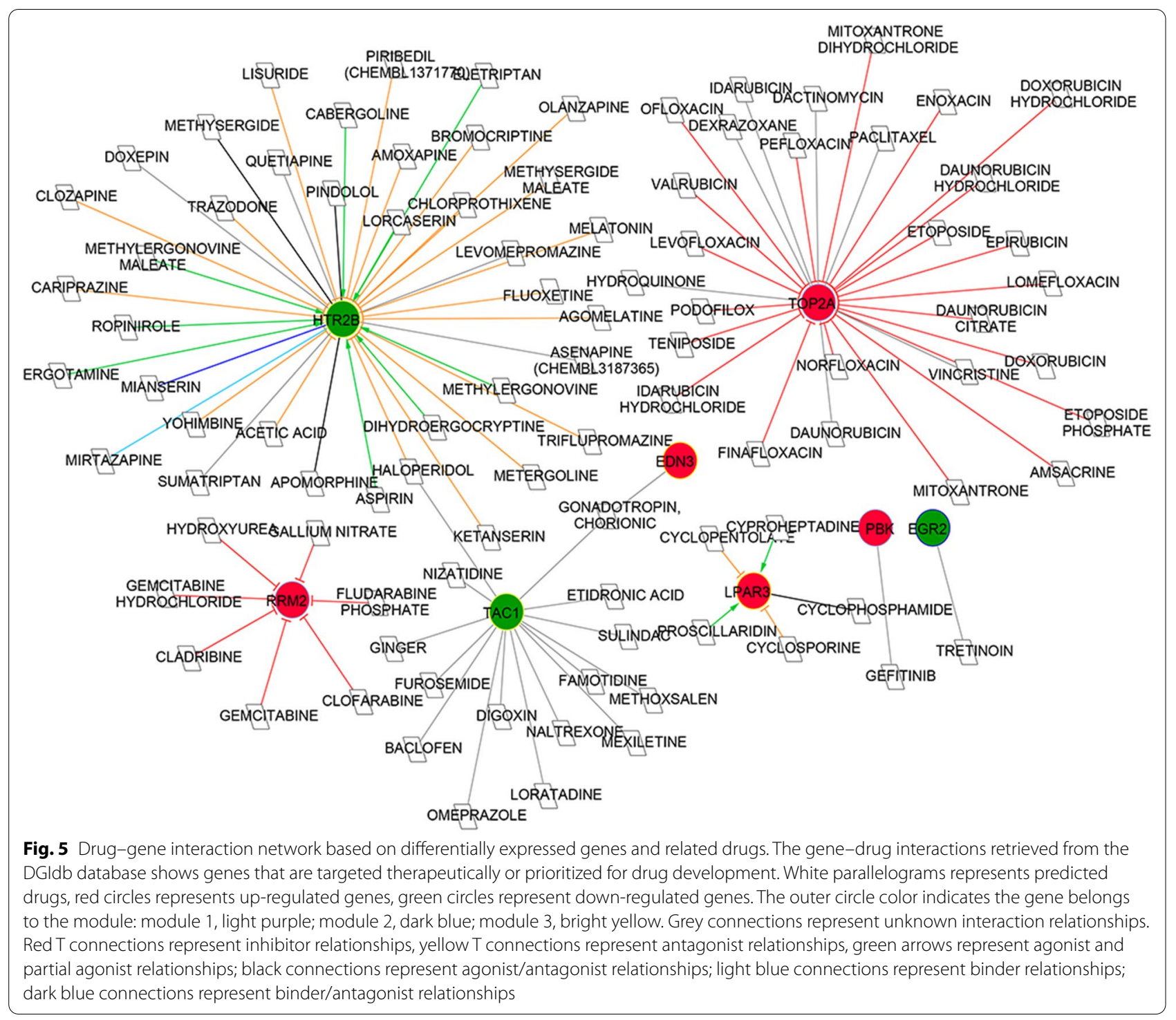

a neoadjuvant treatment for breast cancer [41]. TOP2A was also reported to be a predictive biomarker in endometrial cancer patients receiving taxane-containing adjuvant chemotherapy [42]. In the current study, drug-gene interaction analysis showed that there was a negative association between up-regulated TOP2A and epirubicin. Thus, we speculate that TOP2A might be a crucial target to investigate the potential effect of epirubicin in postmenopausal women. In addition, among the 94 obtained drugs, only 12 drugs were reported to play role in endometrium of in postmenopausal women in studies, of which 7 drugs (for example, epirubicin) were found to target TOP2A. These suggested that TOP2A was a crucial target. Moreover, TOP2A was a significant DEGs in tibolone treated endometrium of postmenopausal women. From all above, we speculate that epirubicin may have potential clinical value in the treatment for endometrium of postmenopausal women. However, the role of epirubicin and its clinical value for endometrium of postmenopausal women should be confirmed by more in vitro and in vivo experiments.

Despite these exciting new findings, there remains some limitations in this study. (1) we preliminarily investigated the DEGs in tibolone treated endometrium of postmenopausal women and their involved biological processes and pathways. The expression of these DEGs should be confirmed in clinical samples by PCR and Western blot, and their potential functions should be investigated by a series of functional experiments. (2) Only nine samples in each group were included. 


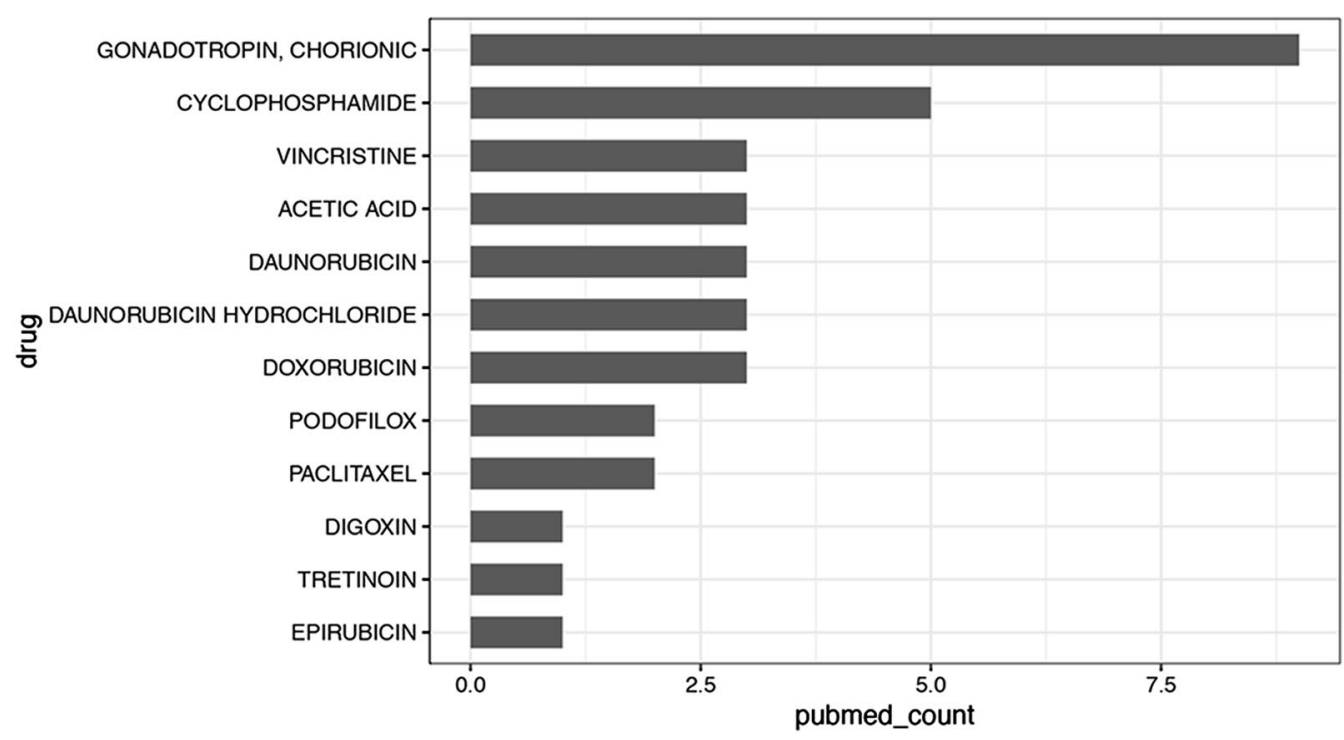

Fig. 6 Novel drug predications based on literature review. Results of a key word search of the PubMed database. Key words included 'postmenopausal' and 'endometrium,' as well as drug names. Drugs reported in 0 to 5 studies relevant to post-menopause and endometrium were considered novel drugs for investigation. The $x$-axis represents the number of published documents; the $y$-axis indicates drug names

Further investigation based on a large sample size was required to investigate the molecular mechanism. (3) Several drugs were predicted to target TOP2A, which was a significant DEGs in tibolone treated endometrium of postmenopausal women. It was needed to further investigate whether TOP2A was a direct target of tibolone in endometrium of postmenopausal women and whether these drugs could replace tibolone to target TOP2A in postmenopausal women.

\section{Conclusion}

In conclusion, expression changes of multiple genes were found in endometrium of tibolone treated postmenopausal women. These genes were implicated in cilium movement, calcium ion homeostasis, regulation of hormone levels and complement/coagulation cascades. CCDC114 and DNAI2, which both participate in cilium movement function, are upregulated in postmenopausal women treated with tibolone. TOP2A might be a crucial target of tibolone in endometrium of postmenopausal women.

\section{Abbreviations}

DEGs: Differentially expressed genes; PPI: Protein-protein interaction network; SLE: Systemic lupus erythematosus; CCDC: Coiled-Coil Domain Containing; DNAI: Dynein Axonemal Intermediate Chain; BP: Biological process; RRM2: Reductase regulatory subunit M2; TOP2A: DNA topoisomerase II A; PBK: PDZ binding kinase.Supplementary information

\section{Supplementary information}

accompanies this paper at https://doi.org/10.1186/s12905-020-01156-6.

Additional file 1: Figure S1. The workflow of this study.

Additional file 2: Table S1. The enriched biological processes terms and KEGG pathways for DEGs.

Additional file 3: Table S2. Results of topological properties analysis for nodes in PPI network.

Additional file 4: Table S3. The enriched GO terms and KEGG pathways for genes in significant modules identified from PPI network.

Additional file 5: Table S4. The obtained drug-gene interactions information from GDIdb database.

Additional file 6: Table S5. Validation of the predictive drugs in Pubmed.

\section{Acknowledgements}

Not applicable.

\section{Authors' contributions}

Conception and design of the research: GH, Yanhua L and Yanqing L; acquisition of data: YW, FK and QP; analysis and interpretation of data: YW, FK and QP; statistical analysis: YW, FK and QP; drafting the manuscript: Yanhua $L$ and Yanqing L; revision of manuscript for important intellectual content: GH. All authors read and approved the final manuscript.

\section{Funding}

Not applicable.

\section{Availability of data and materials}

The datasets used and/or analyzed during the current study are available from the corresponding author on reasonable request.

Ethics approval and consent to participate Not applicable. 


\section{Consent for publication \\ Not applicable.}

\section{Competing interests}

The authors declare that they have no competing interests.

\begin{abstract}
Author details
'Department of Gynecology, Affiliated Hospital of Jining Medical University, Jining 272000, Shandong, China. ${ }^{2}$ Department of General Medicine, Jining No. 1 People's Hospital, Jining 272011, Shandong, China. ${ }^{3}$ Department of Internal Medicine-Cardiovascular, Affiliated Hospital of Taishan Medical University, Taian 271000, Shandong, China. ${ }^{4}$ Laboratory of Developmental and Evolutionary Biology, School of Life Sciences, Shandong University of Technology, Zibo 255049, Shandong, China. ${ }^{5}$ Department of Obstetrics and Gynecology, People's Hospital of Jiaxiang County, No. 188 Yingfeng Road, Jiaxiang, Jining 272400, Shandong, China.
\end{abstract}

Received: 1 November 2019 Accepted: 20 December 2020 Published online: 11 June 2021

\section{References}

1. Luís Â, Domingues F. Effects of red clover on perimenopausal and postmenopausal women's blood lipid profile: a meta-analysis. Climact J Int Menop Soc. 2018;21(5):446-53.

2. Lee SN, Cho JY, Eun YM, Song SW, Moon KW. Associations between osteoporosis and coronary artery disease in postmenopausal women. Climact J Int Menop Soc. 2016;19(5):5.

3. Pinkerton JV. Hormone therapy for postmenopausal women. N Engl J Med. 2020;382(5):446-55.

4. Brinton LA, Trabert B, Anderson GL, Falk RT, Felix AS, Fuhrman BJ, Gass ML, Kuller LH, Pfeiffer RM, Rohan TE. Serum estrogen and estrogen metabolites and endometrial cancer risk among postmenopausal women. Cancer Epidemiol Biomark Prevent. 2016:25(7):1081-9.

5. Grady D, Rubin SM, Petitti DB, Fox CS, Black D, Ettinger B, Ernster VL, Cummings SR. Hormone therapy to prevent disease and prolong life in postmenopausal women. Int J Gynecol Obstet. 1993;42(2):225-6.

6. Hanifi-Moghaddam P, Boers-Sijmons B, Klaassens AH, van Wijk FH, den Bakker MA, Ott MC, Shipley GL, Verheul HA, Kloosterboer HJ, Burger CW, et al. Molecular analysis of human endometrium: short-term tibolone signaling differs significantly from estrogen and estrogen + progestagen signaling. J Mol Med (Berl). 2007;85(5):471-80.

7. Formoso G, Perrone E, Maltoni S, Balduzzi S, Wilkinson J, Basevi V, Marata AM, Magrini N, D'Amico R, Bassi C, Maestri E. Short-term and long-term effects of tibolone in postmenopausal women. Cochrane Database Syst Rev. 2016;10(10):CD008536.

8. Sjögren LL, Mørch LS, Løkkegaard E. Hormone replacement therapy and the risk of endometrial cancer: a systematic review. Maturitas. 2016;91:25-35.

9. Archer DF, Hendrix S, Gallagher JC, Rymer J, Skouby S, Ferenczy A, den Hollander W, Stathopoulos V, Helmond FA. Endometrial effects of tiboIone. J Clin Endocrinol Metab. 2007:92(3):911-8.

10. Huang K-E, Baber R. Updated clinical recommendations for the use of tibolone in Asian women. Climacteric. 2010;13(4):317-27.

11. Reis BF, Lima S, Silva G, Francisco A, Barbosa L, Archangelo S, Grande RM. Effects of low dose of tibolone on steroid receptors and $\mathrm{BCl}-2$ on the postmenopausal endometrium. Histol Histopathol. 2016;31(6):629-34.

12. Hanifi-Moghaddam $P$, Boers-Sijmons B, Klaassens AHA, Wijk FH, Van BMA, Den OMC, Shipley GL, Verheul HAM, Kloosterboer HJ, Burger CW. Molecular analysis of human endometrium: short-term tibolone signaling differs significantly from estrogen and estrogen + progestagen signaling. J Mol Med. 2007;85(5):471-80.

13. Gautier L, Cope L, Bolstad BM, Irizarry RA. affy —analysis of Affymetrix GeneChip data at the probe level. Bioinformatics. 2004;20(3):307-15.

14. Smyth GK. limma: linear models for microarray data. In: Gentleman R, Carey VJ, Huber W, Irizarry RA, Dudoit S, editors. Bioinformatics and computational biology solutions using $\mathrm{R}$ and bioconductor. New York: Springer; 2005. p. 397-420.
15. Huang DW, Sherman BT, Lempicki RA. Systematic and integrative analysis of large gene lists using DAVID bioinformatics resources. Nat Protoc. 2008:4:44.

16. Ashburner M, Ball CA, Blake JA, Botstein D, Butler H, Cherry JM, Davis AP, Dolinski K, Dwight SS, Eppig JT, et al. Gene ontology: tool for the unification of biology. Nat Genet. 2000;25(1):25-9.

17. Kanehisa M, Goto S. KEGG: Kyoto encyclopedia of genes and genomes. Nucleic Acids Res. 2000;28(1):27-30.

18. Szklarczyk D, Franceschini A, Wyder S, Forslund K, Heller D, Huerta-Cepas J, Simonovic M, Roth A, Santos A, Tsafou KP. STRING v10: protein-protein interaction networks, integrated over the tree of life. Nucleic Acids Res 2015; 43(Database issue):D447-52.

19. Yu Tang ML, Wang J, Pan Y, Wu F-X. CytoNCA: a cytoscape plugin for centrality analysis and evaluation of biological networks. BioSystems. 2014;11:005.

20. Bader GD, Hogue CW. An automated method for finding molecular complexes in large protein interaction networks. BMC Bioinform. 2003;4:2.

21. Cotto KC, Wagner AH, Feng YY, Kiwala S, Coffman AC, Spies G, Wol$\operatorname{lam}$ A, Spies NC, Griffith OL, Griffith M.: DGldb 3.0: a redesign and expansion of the drug-gene interaction database. Nucleic Acids Res. 2018;46(D1):D1068-73.

22. Shang W, Burton JC, Behringer RR, Larina IV.: Functional optical coherence tomography for high-resolution mapping of cilia beat frequency in the mouse oviduct in vivo. In: Photonic therapeutics and diagnostics XII: 2016; 2016.

23. Bylander A, Lind K, Goksör M, Billig H, Larsson DJ. The classical progesterone receptor mediates the rapid reduction of fallopian tube ciliary beat frequency by progesterone. Reprod Biol Endocrinol. 2013;11(1):33.

24. Haider S, Gamperl M, Burkard TR, Kunihs V, Kaindl U, Junttila S, Fiala C, Schmidt K, Mendjan S, Knöfler M. Estrogen signaling drives ciliogenesis in human endometrial organoids. Endocrinology. 2019;160(10):2282-97.

25. McClung EC, Berglund A, Welsh E, Xiong Y, Robertson S, Boac B, Chon HS, Magliocco A, Marchion D. Differential expression of cilia-related genes in Endometrioid endometrial carcinoma: prognostic implications and therapeutic opportunities. Gynecol Oncol. 2016;143(1):201.

26. Gur M, Cohen EB, Genin O, Fainsod A, Perles Z, Cinnamon Y. Roles of the cilium-associated gene CCDC11 in left-right patterning and in laterality disorders in humans. Int J Dev Biol. 2017:61(3-4-5):267.

27. Milla C. Exome sequencing identifies mutations in CCDC114 as a cause of primary ciliary dyskinesia. Am J Hum Genet. 2013;92(1):99-106.

28. PaffT, Onoufriadis A, Anthony D, Shoemark A, Micha D, Kuyt B, Schmidts M, Petridi S, Dankert-Roelse JE, Haarman EG. Mutation in the CCDC114 gene causes primary ciliary dyskinesia with normal fertility in the isolated Volendam population. Tijdschrift Voor Kindergeneeskunde. 2013;81(1):92-92

29. Loges NT, Olbrich H, Fenske L, Mussaffi H, Horvath J, Fliegauf M, Kuhl H, Baktai G, Peterffy E, Chodhari R. DNAI2 mutations cause primary ciliary dyskinesia with defects in the outer dynein arm. Am J Hum Genet. 2008;83(5):547-58

30. Shorter SL, Albaghdadi AJH, Kan FWK. Alterations in oviductal cilia morphology and reduced expression of axonemal dynein in diabetic NOD mice. Tissue Cell. 2016;48(6):588-95.

31. Zhaojuan Y, Ji W. Mouse dynein axonemal intermediate chain 2: cloning and expression. DNA Cell Biol. 2008;27(9):479-88.

32. Ganzina F. 4'-epi-doxorubicin, a new analogue of doxorubicin: a preliminary overview of preclinical and clinical data. Cancer Treat Rev. 1983;10(1):1-22.

33. Cersosimo RJ, Hong WK. Epirubicin: a review of the pharmacology, clinical activity, and adverse effects of an adriamycin analogue. J Clin Oncol. 1986:4(3):425-39.

34. Feher O, Vodvarka P, Jassem J, Morack G, Advani SH, Khoo KS, Doval DC, Ermisch S, Roychowdhury D, Miller MA. First-line gemcitabine versus epirubicin in postmenopausal women aged 60 or older with metastatic breast cancer: a multicenter, randomized, phase III study. Ann Oncol. 2005;16(6):899-908.

35. Bastholt L, Dalmark M, Gjedde SB, Pfeiffer P, Pedersen D, Sandberg E, Kjaer $M$, Mouridsen HT, Rose C, Nielsen OS. Dose-response relationship of epirubicin in the treatment of postmenopausal patients with metastatic breast cancer: a randomized study of epirubicin at four different dose levels performed by the Danish Breast Cancer Cooperative Group. J Clin Oncol. 1996:14(4):1146-55. 
36. Coombes RC, Bliss JM, Espie M, Erdkamp F, Wals J, Tres A, Marty M, Coleman RE, Tubianamathieu N, den Boer MO. Randomized, phase III trial of sequential epirubicin and docetaxel versus epirubicin alone in postmenopausal patients with node-positive breast cancer. J Clin Oncol. 2011;29(24):3247-54.

37. Wils JA, Bliss JM, Marty M, Coombes G, Fontaine C, Morvan F, Olmos T, Perez-Lopez FR, Vassilopoulos P, Woods E. Epirubicin plus tamoxifen versus tamoxifen alone in node-positive postmenopausal patients with breast cancer: a randomized trial of the International Collaborative Cancer Group. J Clin Oncol. 1999;17(7):1988-98.

38. Lehne G, Deangelis $\mathrm{P}$, Clausen $\mathrm{O}$, Hall K, Huitfeldt $H$, Rugstad H. Pharmacokinetics and cytotoxicity of epirubicin (epi) in drug-resistant human hepatoma-cells (hb8065). Int J Oncol. 1994;4(6):1229-35.

39. Tarpgaard LS, Qvortrup C, Nygård SB, Nielsen SL, Andersen DR, Jensen NF, Stenvang J, Detlefsen S, Brünner N, Pfeiffer P. A phase II study of Epirubicin in oxaliplatin-resistant patients with metastatic colorectal cance and TOP2A gene amplification. Bmc Cancer. 2016;16(1):91.
40. Knoop A, Knudsen H, Balslev E, Rasmussen B, Overgaard J, Nielsen K, Schønau A, Gunnarsdóttir K, Mouridsen H, Ejlertsen B. 674 Topoisomerase II alpha (TOP2A) alterations as a predictive marker for epirubicin sensitivity in 805 high-risk breast cancer patients. A randomised DBCG trial (DBCG89D). Eur J Cancer Suppl. 2003;1(5):S202-3.

41. Erica M, Christine D, Chiara B, Regan MM, Catherine O, Denis L, Francesca G, Martine PG, Christos S, Rimm DL. TOP2A protein by quantitative immunofluorescence as a predictor of response to epirubicin in the neoadjuvant treatment of breast cancer. Future Oncol. 2013;9(10):1477-87.

42. Ito F, Furukawa N, Nakai T. Evaluation of TOP2A as a predictive marker for endometrial cancer with taxane-containing adjuvant chemotherapy. Int J Gynecol Cancer. 2016;26(2):325-30.

\section{Publisher's Note}

Springer Nature remains neutral with regard to jurisdictional claims in published maps and institutional affiliations.
Ready to submit your research? Choose BMC and benefit from:

- fast, convenient online submission

- thorough peer review by experienced researchers in your field

- rapid publication on acceptance

- support for research data, including large and complex data types

- gold Open Access which fosters wider collaboration and increased citations

- maximum visibility for your research: over $100 \mathrm{M}$ website views per year

At BMC, research is always in progress.

Learn more biomedcentral.com/submissions 